\title{
A converse Lyapunov theorem for almost sure stabilizability *
}

\author{
Annalisa Cesaroni \\ Dipartimento di Matematica P. e A. \\ Università di Padova \\ via Belzoni 7, 35131 Padova, Italy \\ acesar@math.unipd.it
}

\begin{abstract}
We prove a converse Lyapunov theorem for almost sure stabilizability and almost sure asymptotic stabilizability of controlled diffusions: given a stochastic system a.s. stochastic open loop stabilizable at the origin, we construct a lower semicontinuous positive definite function whose level sets form a local basis of viable neighborhoods of the equilibrium. This result provides, with the direct Lyapunov theorems proved in a companion paper, a complete Lyapunov-like characterization of the a.s. stabilizability.

Key words. Degenerate diffusion, almost sure stability, stabilizability, asymptotic stability, stochastic control, control Lyapunov function, viscosity solutions, Hamilton-Jacobi-Bellman inequalities, viability.
\end{abstract}

\section{Introduction}

In this paper we provide a Lyapunov characterization of almost sure stochastic open loop stability at an equilibrium of controlled diffusion processes in $\mathbb{R}^{N}$

$$
(C S D E)\left\{\begin{array}{l}
d X_{t}=f\left(X_{t}, \alpha_{t}\right) d t+\sigma\left(X_{t}, \alpha_{t}\right) d B_{t}, \alpha_{t} \in A, t>0 \\
X_{0}=x
\end{array}\right.
$$

This notion has been introduced in a companion paper $[6$ by Bardi and the author (see also [5]): we say that $(C S D E)$ is a.s. (open loop) stabilizable if for any $\eta>0$ there exists $\delta>0$ such that, for any $x$ with $|x| \leq \delta$, there exists $\alpha$ such that the corresponding process satisfies $\left|X_{t}\right| \leq \eta$ for all $t \geq 0$ almost surely. If, in addition, the trajectory is asymptotically approaching a.s. the equilibrium, we say the system is a.s. (open loop) asymptotically stabilizable. The definitions imply in particular that these properties are never verified by nondegenerate processes. This stochastic stability describes a behaviour very similar to a stable deterministic system and is stronger than pathwise stability and stability in probability (see [17, 19, 21]). We characterize it by means of appropriate control Lyapunov functions. These functions have been introduced in [5] and are lower semicontinuous (LSC), continuous at the equilibrium, positive definite, proper. Moreover they satisfy the following infinitesimal decrease condition:

$$
\sup _{\sigma(x, \alpha)^{T} D V(x)=0}\left\{-D V(x) \cdot f(x, \alpha)-\operatorname{trace}\left[a(x, \alpha) D^{2} V(x)\right]\right\} \geq l(x),
$$

where $a:=\sigma \sigma^{T} / 2, l \equiv 0$ for Lyapunov functions and positive definite for strict Lyapunov functions. This is not a standard Hamilton-Jacobi-Bellman inequality, because the constraint on the controls

${ }^{*}$ This research was partially supported by M.I.U.R., project "Viscosity, metric, and control theoretic methods for nonlinear partial differential equations", and by GNAMPA-INDAM, project "Partial differential equations and control theory". 
depends on $V$ : we are allowing diffusion only in the directions tangential to the sublevel sets of $V$. If we eliminate this constraint, the differential inequality which is left is the infinitesimal decrease condition on Lyapunov functions for the stability in probability (see [12, 11]). We prove that $V$ satisfies (1) if and only if it satisfies the following monotonicity condition:

$$
\forall x \exists \alpha: \quad \sup _{t \geq 0} \operatorname{ess} \sup _{\omega \in \Omega}\left(V\left(X_{t}\right)+\int_{0}^{t} l\left(X_{s}\right) d s\right) \leq V(x)
$$

where the essential supremum is intended with respect to the probability measure $\mathbf{P}_{x}$. This means in particular that the process $V\left(X_{t}\right)$ is a positive supermaxingale according to the definition given in 10]: this is the natural counterpart of the requirement on the process $V\left(X_{t}\right)$ to be a positive supermartingale in the context of stability in probability. The monotonicity condition says that the sublevel sets $K_{\mu}:=\{x \quad V(x) \leq \mu\}$ are viable (or weakly invariant) with respect to (CSDE) in the sense that $\forall x \in K_{\mu} \exists \alpha$ such that $X_{t} \in K_{\mu}$ forever almost surely. One of the main tool used in this paper is the geometric Nagumo-type characterization of viability proved recently by Bardi and Jensen in [9] (see also [8], 2] and the references therein for earlier related results).

In [6], Bardi and the author show that the existence of a Lyapunov function (respectively, of a strict Lyapunov function) implies the a.s. stabilizability (respectively, the a.s. asymptotic stabilizability) of the system to the equilibrium. As a simple example of application of this theory, we consider a radial function $V(x)=v(|x|)$, for some real smooth function $v$ with $v^{\prime}(r)>0$ for $r>0$. The system (CSDE) admits $V$ as Lyapunov function if

$$
\forall x \exists \alpha: \sigma(x, \alpha) \cdot x=0 \quad f(x, \alpha) \cdot x+\text { trace } a(x, \alpha) \leq 0 .
$$

Therefore the following conditions are sufficient for the a.s. stabilizability: the radial component of the diffusion is null and its rotational component, which still plays a destabilizing role since trace $a(x, \alpha) \geq 0$, must be compensated by a negative radial component of $f$.

In this paper we prove that the existence of a Lyapunov function is also a necessary condition for a.s. stabilizability (and also for a.s. Lagrange stabilizability in the global case). A Lyapunov function for the system can be defined as

$$
V(x):=\inf \left\{r \mid \exists \bar{\alpha} \text { admissible control such that }\left|\bar{X}_{t}\right| \leq r \text { almost surely } \forall t \geq 0\right\},
$$

or equivalently as

$$
V(x):=\inf _{\alpha \in \mathcal{A}_{x}} \sup _{t \geq 0} \operatorname{ess} \sup _{\omega \in \Omega}\left|X_{t}^{\alpha}\right| .
$$

We prove that this function is LSC, continuous at the origin, positive definite, proper, and satisfies the infinitesimal decrease condition (10) with $l \equiv 0$. For an a.s. asymptotic stabilizable systems in a bounded set $\mathcal{O}$, we build a positive definite Lipschitz continuous function $l$, related to the rate of decrease of the stable trajectories to the equilibrium, by the formula

$$
V(x):=\inf _{\alpha \in \mathcal{A}_{x}} \operatorname{ess} \sup _{\omega \in \Omega} \int_{0}^{+\infty} l\left(X_{t}^{\alpha}\right) d t .
$$

We show that $V$ is finite, LSC, continuous at the origin, positive definite, proper, and satisfies the infinitesimal decrease condition (11).

In both cases the Lyapunov functions are worst-case value function of an appropriate stochastic optimal control problem: we minimize the worst possible cost over all possible paths. This is quite natural since these functions characterize a very strong stability notion. The link between worstcase value functions and viscosity solutions to geometric second order partial differential equations has been recently treated by Soner and Touzi in [25] (see also [10]). They considered stochastic target problems where the controller tries to steer almost surely a controlled process into a given target by judicial choices of controls. The interest in this kind of stochastic control problems in the almost sure setting comes from the relationship with mean curvature type geometric flows and from the applications to the super-replication problems in financial mathematics. Moreover the 
a.s. stability of control systems affected by disturbances modelled as $M$-dimensional white noise is related to the so-called worst-case stability (or robust stability) of deterministic control systems affected by disturbances modelled as (deterministic) $L^{\infty}$ functions with values in $\mathbb{R}^{M}$ (see [16]). The Lyapunov characterization of these two stability properties seems to be an useful tool to give a precise proof of this relationship (see [12]), while a direct proof based on the estimates among the trajectories of the two systems should be rather hard. An approach of this type has been used recently by Da Prato and Frankowska in 14 to prove the equivalence between the invariance with respect to a controlled stochastic system and the invariance with respect to a deterministic system with two (non competitive) controls.

We conclude with some additional references on converse Lyapunov theorems. For controlled deterministic systems, there are theorems characterizing the stochastic open loop stabilizability by means of LSC appropriate Lyapunov functions (see [3]). Soravia in [28] showed that the stability at an equilibrium is equivalent to the continuity at such point of the value function $V(x)=\inf _{\alpha \in \mathcal{A}_{x}} \sup _{t \geq 0} U\left(X_{t}\right)$ where the level sets of $U$ form a local basis of neighborhoods of the equilibrium. For asymptotically controllable systems, Sontag and Sussmann ([26, 27]) provided a characterization of asymptotic controllability by means of continuous Lyapunov functions such as $V(x)=\inf _{\alpha} \int_{0}^{+\infty} l\left(X_{t}\right) d t$ where $l$ is an appropriate positive definite function. Recently Rifford (23]) proved a converse Lyapunov theorem in the framework of Lipschitz continuous functions which are semiconcave outside the equilibrium. In the stochastic setting, Has'minskii (17, 19]) obtained a converse theorem for stability in probability of uncontrolled diffusion processes, strictly nondegenerate outside the equilibrium, by means of $\mathcal{C}^{2}$ Lyapunov functions, using the Maximum Principle and the properties of solutions of uniformly elliptic equation. Kushner proved in [20] a characterization of asymptotic uniform stochastic stability by means of continuous Lyapunov functions (here, however, the infinitesimal decrease condition is given in terms of the weak generator of the process). In the forthcoming paper [11] (see also [12]) the author extends the direct Lyapunov method by Has'minskii and Kushner to the study of stochastic open loop stabilizability in probability in terms of merely semicontinuous Lyapunov functions which satisfy in the viscosity sense an appropriate infinitesimal decrease condition and provides also in this setting converse Lyapunov theorems.

The paper is organized as follows. In Section 2 we give the definition of stochastic open loop a.s. stabilizability, in Section 3 we introduce the appropriate concept of Lyapunov function for the study of such stability. Section 4 is devoted to the viability properties of sublevel sets of Lyapunov functions. Section 5 contains the main results: the converse Lyapunov theorems. Finally in Section 6 we give the extension to general equilibrium sets.

\section{Almost sure Lyapunov stabilizability}

We consider a controlled Ito stochastic differential equation:

$$
(C S D E)\left\{\begin{array}{l}
d X_{t}=f\left(X_{t}, \alpha_{t}\right) d t+\sigma\left(X_{t}, \alpha_{t}\right) d B_{t}, t>0 \\
X_{0}=x
\end{array}\right.
$$

where $B_{t}$ is an $M$-dimensional Brownian motion. Throughout the paper we assume that $f, \sigma$ are continuous functions defined in $\mathbb{R}^{N} \times A$, where $A$ is a compact metric space, which take values, respectively, in $\mathbb{R}^{N}$ and in the space of $N \times M$ matrices, and satisfying for all $x, y \in \mathbb{R}^{N}$ and all $\alpha \in A$

$$
|f(x, \alpha)-f(y, \alpha)|+\|\sigma(x, \alpha)-\sigma(y, \alpha)\| \leq C|x-y|,
$$

We define $a(x, \alpha):=\frac{1}{2} \sigma(x, \alpha) \sigma(x, \alpha)^{T}$ and assume

$$
\{(a(x, \alpha), f(x, \alpha)): \alpha \in A\} \quad \text { is convex for all } x \in \mathbb{R}^{N} .
$$

The class of admissible controls is the class of strict controls, as defined in [18, Definition 2.2]: they are $A$ valued, progressively measurable processes $\alpha_{t}$ such that there exists a solution $X_{t}^{\alpha}$ to $(C S D E) . \mathcal{A}_{x}$ denotes the class of admissible control for a given initial datum $x$, with $\alpha$. its generic 
element (although it is not a standard function $\mathbb{R} \rightarrow A$ ), and with $X$. the corresponding solution of $(C S D E)$. We recall also a theorem on the existence of optimal control for stochastic control problems.

Theorem 1 (Theorem 4.7 and Corollary 4.8 [18]). Under the convexity assumption (4), for every initial data $x \in \mathbb{R}^{N}$ there exists an admissible control realizing the minimum in the control problem $\inf _{\alpha} \mathbf{E} J(x, \alpha)$ where the cost functional $J(x, \alpha)$ satisfies standard regularity assumptions.

We state now the definition of almost sure stochastic open loop stabilizability, which has been introduced and studied in the paper [6] (see also [5] for the uncontrolled case). We introduce the classes of comparison functions.

Definition 2 (comparison functions). $\mathcal{K}$ denotes the class of real continuous functions $\gamma$ strictly increasing and such that $\gamma(0)=0 ; \mathcal{K}_{\infty}$ contains the functions $\gamma \in \mathcal{K}$ such that $\lim _{r \rightarrow+\infty} \gamma(r)=$ $+\infty$. Finally $\mathcal{K} \mathcal{L}$ denotes the class of continuous functions $\beta: \mathbb{R} \times \mathbb{R} \rightarrow \mathbb{R}$ which are strictly increasing in the first variable, strictly decreasing in the second variable, which satisfy $\beta(0, t)=0$ for $t \geq 0, \lim _{t \rightarrow+\infty} \beta(r, t)=0$ for $r \geq 0$.

Definition 3 (a.s. stabilizability). The system $(C S D E)$ is almost surely (open-loop Lyapunov) stabilizable at the origin if there exists $\gamma \in \mathcal{K}$ and $\delta_{o}>0$ such that for any starting point $x$ with $|x| \leq \delta_{o}$

$$
\exists \bar{\alpha} . \in \mathcal{A}_{x}: \quad\left|\bar{X}_{t}\right| \leq \gamma(|x|) \quad \forall t \geq 0 \text { a.s. }
$$

If $\gamma$ can be chosen in $\mathcal{K}_{\infty}$ and the estimate (5) holds in the whole space $\mathbb{R}^{N}$, the system is also almost surely (open-loop) Lagrange stabilizable, or it has the property of uniform boundedness of trajectories.

Remark 4. We could define the a.s. stabilizability equivalently as follows:

the system is a.s. (open loop) stabilizable at the origin if for every $\varepsilon>0$ there exists $\delta>0$ such that for $|x| \leq \delta$ there exists an admissible control function $\bar{\alpha} . \in \mathcal{A}_{x}$ whose corresponding trajectory $\bar{X}$. verifies $\left|\bar{X}_{t}\right| \leq \varepsilon$ for all $t \geq 0$ almost surely.

Remark 5. A necessary condition for the a.s. stabilizability at the origin is that the origin is a controlled equilibrium of $(C S D E)$, i.e.,

$$
\exists \bar{\alpha} \in A: f(0, \bar{\alpha})=0, \sigma(0, \bar{\alpha})=0 .
$$

Definition 6 (a.s. asymptotic stabilizability). The system (CSDE) is almost surely (open loop) locally asymptotically stabilizable (or a. s. locally asymptotically controllable) at the origin if there is $\beta \in \mathcal{K} \mathcal{L}$ and $R>0$ such that for any starting point $x$ with $|x| \leq R$ there exists $\bar{\alpha} . \in \mathcal{A}_{x}$

$$
\left|\bar{X}_{t}\right| \leq \beta(|x|, t) \quad \forall t \geq 0 \text { a.s. }
$$

\section{Lyapunov functions for a.s. stabilizability}

In this section we introduce the appropriate concept of Lyapunov function for the study of the almost sure stochastic stability.

We recall the definition of the second order semijet (see [13) of a LSC function $V$ at $x \mathcal{J}^{2,-} V(x):=$ $\left\{(p, Y) \in \mathbb{R}^{N} \times S(N)\right.$ such that for $\left.y \rightarrow x, V(y) \geq V(x)+p \cdot(y-x)+\frac{1}{2}(y-x) \cdot Y(y-x)+o\left(|y-x|^{2}\right)\right\}$.

Definition 7 (control Lyapunov function). Let $\mathcal{O} \subseteq \mathbb{R}^{N}$ be an open set containing the origin. A function $V: \mathcal{O} \rightarrow[0,+\infty)$ is a control Lyapunov function for the a.s. stabilizability of (CSDE) if

(i) $V$ is lower semicontinuous and continuous at 0 ;

(ii) $V$ is positive definite, i.e., $V(0)=0$ and $V(x)>0$ for all $x \neq 0$;

(iii) $V$ is proper, i.e., the sublevel sets $\{x \mid V(x) \leq \mu\}$ are bounded $\forall \mu \in[0, \infty)$; 
(iv) $V$ is a viscosity supersolution in $\mathcal{O} \backslash\{0\}$ of the equation:

$$
\sup _{\sigma(x, \alpha) \cdot D V(x)=0}\left\{-D V(x) \cdot f(x, \alpha)-\operatorname{trace}\left[a(x, \alpha) D^{2} V(x)\right]\right\} \geq 0,
$$

in the following sense: for all $x \in \mathcal{O} \backslash\{0\}$ and $(p, Y) \in \mathcal{J}^{2,-} V(x)$ there exists $\bar{\alpha} \in A$ :

$$
\sigma(x, \bar{\alpha})^{T} p=0 \quad \text { and } \quad-p \cdot f(x, \bar{\alpha})-\operatorname{trace}[a(x, \bar{\alpha}) Y] \geq 0 .
$$

If there exists a positive definite, Lipschitz continuous $l: \mathcal{O} \rightarrow \mathbb{R}$ such that

$$
\sup _{\sigma(x, \alpha) \cdot D V(x)=0}\left\{-D V(x) \cdot f(x, \alpha)-\operatorname{trace}\left[a(x, \alpha) D^{2} V(x)\right]\right\} \geq l(x)
$$

then $V$ is a strict control Lyapunov function for the a.s. stabilizability of (CSDE).

The inequality (8) is not the standard Hamilton-Jacobi-Bellman inequality arising in stochastic optimal control. We have an implicit constraint on the controls, $\sigma(x, \alpha) \cdot D V(x)=0$, i.e. depending on the generalized subgradients of the solution: we are allowing only controls which render the diffusion matrix tangential in some generalized sense to the sublevel sets of $V$. This implies that the diffusion has to degenerate in a large set, for some control.

Because of this constraint, though, the nonlinearity

$$
F(x, p, X)=\sup \{-p \cdot f(x, \alpha)-\operatorname{trace}[a(x, \alpha) X] \mid a \in A \sigma(x, \alpha) \cdot p=0\}
$$

is geometric in the sense that it satisfies the following rescaling property $F(x, \lambda p, \lambda X+\mu p \otimes p)=$ $\lambda F(x, p, X)$ for every $\lambda>0$ and $\mu \in \mathbb{R}$, where $p \otimes p$ is the $N \times N$ matrix whose $(i, j)$ entry is $p_{i} p_{j}$. This permits to prove the following lemma on the change of unknown (for the proof see [6], 12]).

Lemma 8. Assume that $v$ is a LSC viscosity supersolution of equation (8) in an open set $\mathcal{O}$. Let $\phi$ be a twice continuously differentiable strictly increasing real map. Then $w=\phi \circ v$ is still a viscosity supersolution of equation (8) in $\mathcal{O}$.

\section{Viability properties of Lyapunov functions}

We study now a viability property of the sublevel sets of viscosity supersolution of the nonstandard Hamilton-Jacobi-Bellman inequality (8). We recall the definition of almost sure viability (named also controlled invariance and weak invariance) of an arbitrary closed set for a controlled diffusion process.

Definition 9 (viable set). A closed set $K \subset \mathbb{R}^{N}$ is viable or controlled invariant or weakly invariant for the stochastic system (CSDE) if for all initial points $x \in K$ there exists an admissible control $\alpha . \in \mathcal{A}_{x}$ such that the corresponding trajectory $X$. satisfies $X_{t} \in K$ for all $t>0$ almost surely.

This property was studied by Aubin and Da Prato [2] and, more recently, by Bardi and Jensen [9]. The main result of [9] is the equivalence between the viability of a closed set $K$ and a Nagumotype geometric condition. This geometric condition is given in terms of the second order normal cone to a closed set $K \subset \mathbb{R}^{N}$, first introduced in $[$,

$$
\begin{aligned}
\mathcal{N}_{K}^{2}(x):= & \left\{(p, Y) \in \mathbb{R}^{N} \times S(N): \text { for } y \rightarrow x, y \in K,\right. \\
& \left.p \cdot(y-x)+\frac{1}{2}(y-x) \cdot Y(y-x) \geq o\left(|y-x|^{2}\right)\right\}
\end{aligned}
$$

where $S(N)$ is the set of symmetric $N \times N$ matrices. Note that, if $\partial K$ is a smooth surface in a neighborhood of $x, p /|p|$ is the interior normal and $Y$ is related to the second fundamental form of $\partial K$ at $x$, see $[8]$. 
Theorem 10 (Viability theorem [9]). Assume conditions (3) and 4). Then $K$ is viable for $(C S D E)$ if and only if

$$
\forall x \in \partial K, \forall(p, Y) \in \mathcal{N}_{K}^{2}(x), \exists \alpha \in A: \quad f(x, \alpha) \cdot p+\operatorname{trace}[a(x, \alpha) Y] \geq 0 .
$$

Moreover, for the same $\alpha$ we have that $\sigma(x, \alpha) \cdot p=0$.

Using this result we obtain the following characterization, which is a variant of a result contained in [6] (see also [5]).

Lemma 11. Assume conditions (3) and (4). Consider an open set $\mathcal{O} \subseteq \mathbb{R}^{N}$ and a LSC function $V: \mathcal{O} \rightarrow \mathbb{R}$. If $V$ is a viscosity supersolution of

$$
\sup _{\sigma(x, \alpha) \cdot D V=0}\left\{-D V \cdot f(x, \alpha)-\operatorname{trace}\left[a(x, \alpha) D^{2} V\right]\right\} \geq 0
$$

in $\mathcal{O}$, then the sublevel sets $\{V(x) \leq \mu\}$ whose boundary is entirely contained in $\mathcal{O}$ are viable with respect to (CSDE). Viceversa, suppose $\bar{\mu}$ is the maximal value for which the sublevel set $\{V(x) \leq \mu\}$ has boundary entirely contained in $\mathcal{O}$. Then the function $\bar{V}(x):=V(x) \wedge \bar{\mu}$ is a viscosity supersolution of (10) in $\mathcal{O}$.

Observe that if $\mathcal{O}=\mathbb{R}^{N}$, every sublevel set of $V$ is closed (in particular we can take $\bar{\mu}=+\infty$ ).

Proof. For every $\mu \leq \bar{\mu}, K_{\mu}:=\{x \mid V(x) \leq \mu\}$. We define now the LSC function $V_{\mu}(x):=\mu$ for $x \in K_{\mu}$ and $+\infty$ elsewhere. From the definitions, it is easy to check that $\mathcal{J}^{2,-} V_{\mu}(x)=$ $-\mathcal{N}_{K}^{2}(x), \quad \forall x \in \partial K_{\mu}$, so, by the Viability Theorem 10] $V_{\mu}$ is a viscosity supersolution of (10) if and only if $K_{\mu}$ is viable.

We assume that $V$ is a viscosity supersolution of (10). Now for $\lambda>0$ fixed, we define the nondecreasing continuous real function

$$
\psi_{\lambda}(t)= \begin{cases}\mu, & t \leq \mu, \\ \lambda^{2}(t-\mu)+\mu, & \mu \leq t \leq \mu+\frac{1}{\lambda}, \\ \lambda+\mu & t \geq \mu+\frac{1}{\lambda}\end{cases}
$$

The function $\psi_{\lambda} \circ V$ is a viscosity supersolution of equation (10) in $\mathcal{O}$ for every $\lambda$. To prove this fact, we choose a sequence $\psi_{n}$ of strictly increasing, smooth real maps that converge uniformly on compact sets to $\psi_{\lambda}$. Then, for every $n$, the map $\psi_{n} \circ V$ is a viscosity supersolution of equation (10) in $\mathcal{O}$ by Lemma 8 . This permits to conclude, by the stability of viscosity supersolutions with respect to uniform convergence. Next we observe that the net $\psi_{\lambda} \circ V$ is increasing and converges as $\lambda \rightarrow+\infty$ to $V_{\mu}$. Viscosity supersolutions are stable with respect to the pointwise increasing convergence (see [4]). Therefore the indicator function $V_{\mu}$ of $K_{\mu}$ is a viscosity supersolution of equation (10) and then $K_{\mu}$ is viable for $\mu \leq \bar{\mu}$.

Conversely, we assume now that $K_{\mu}$ is viable for every $\mu \leq \bar{\mu}$. Moreover, we observe that

$$
\bar{V}(x)=\inf _{\mu \leq \bar{\mu}} V_{\mu}(x) \wedge \bar{\mu}=\inf \{\mu \leq \bar{\mu} \mid V(x) \leq \mu\} \wedge \bar{\mu} .
$$

So, by the stability properties of viscosity supersolutions, if $V_{\mu}$ solves (10) for every $\mu \leq \bar{\mu}$, then $\bar{V}$ solves (10) too.

This lemma provides the main tool to prove the direct Lyapunov theorems (see [6], [12]).

Theorem 12 (Direct Lyapunov theorem). Assume (3), (4). If the system admits a Lyapunov function in an open set $\mathcal{O}$ containing the equilibrium then

(i) the system $(C S D E)$ is almost surely stabilizable at the origin;

(ii) if, in addition, the domain $\mathcal{O}$ can be chosen as $\mathbb{R}^{N}$, the system is also a.s. Lagrange stabilizable and for all $x \in \mathbb{R}^{N}$ there exists $\bar{\alpha} . \in \mathcal{A}_{x}$ such that the corresponding trajectory $\bar{X}$. satisfies

$$
\left|\bar{X}_{t}\right| \leq \gamma_{1}^{-1}\left(\gamma_{2}(|x|)\right) \quad \forall t \geq 0 \quad \text { a.s. }
$$


with $\gamma_{1}, \gamma_{2} \in \mathcal{K}_{\infty}$.

Assume moreover that $V$ is a strict Lyapunov function then

(i) the system $(C S D E)$ is a.s. locally asymptotically stabilizable at the origin;

(ii) if, in addition, the domain $\mathcal{O}$ of $V$ is all $\mathbb{R}^{N}$, the system is a.s. globally asymptotically stabilizable.

\section{Converse Lyapunov theorems for a.s. stabilizability}

In this section we prove the main results in the article: we assume that the system (CSDE) satisfies an a.s. stabilizability property and construct an appropriate Lyapunov function.

Theorem 13 (a.s. stabilizability). Assume (3), (4). Then

(i) if the system (CSDE) is almost surely stabilizable at the origin in the ball $B_{K}$, the function

$$
V(x)=\left[\inf _{\alpha \in \mathcal{A}_{x}} \sup _{t \geq 0} \operatorname{ess} \sup _{\omega \in \Omega}\left|X_{t}^{\alpha}\right|\right] \wedge K
$$

is a Lyapunov function for the system in $B_{K}$;

(ii) if the system is also a.s. Lagrange stabilizable then the function

$$
V(x)=\inf _{\alpha \in \mathcal{A}_{x}} \sup _{t \geq 0} \text { ess } \sup _{\omega \in \Omega}\left|X_{t}^{\alpha}\right|
$$

is a global Lyapunov function for the system.

Proof. We start proving (i). We start constructing the larger viable set containing the origin in the ball $\overline{B_{K}}$. We construct a nonnegative, uniformly continuous, radial function $c_{K}$ such that $c_{K}(|s|)=0$ if $|s| \leq K$ and $0<c_{K}(|s|) \leq|s|$ for $|s|>K$ and for $\lambda<0$ we consider the value function

$$
W_{K}(x)=\inf _{\alpha} \mathbf{E}_{x} \int_{0}^{+\infty} c_{K}\left(\left|X_{s}^{\alpha}\right|\right) e^{-\lambda s} d s .
$$

It is well known ([22] and [15]) that $W_{K}$ is a continuous viscosity supersolution of the HamiltonJacobi-Bellman equation in $\mathbb{R}^{N}$

$$
\max _{\alpha \in A}\left\{-D W(x) \cdot f(x, \alpha)-\operatorname{trace}\left[a(x, \alpha) D^{2} W(x)\right]\right\}+\lambda W(x) \geq c_{K}(|x|)
$$

The function $W_{K}$ is nonnegative in $\mathbb{R}^{N}$ and $W_{K}(0)=0$ since the origin is a controlled equilibrium as remarked in (6). We consider the propagation set of the minimum value 0 :

$$
\operatorname{Prop}\left(0, W_{K}\right)=\left\{x \in \mathbb{R}^{N} \mid W_{K}(x)=0\right\}=\left\{x \mid \exists \bar{\alpha}: \bar{X}_{t} \in \overline{B_{K}} \text { a.s. } \forall t\right\} \subseteq \overline{B_{K}} .
$$

This set is clearly closed and it can be proved that it is also viable. This follows immediately from Theorem 4.6 in Bardi, Da Lio [7] or can be checked directly.

The candidate Lyapunov function is defined in the set $\overline{\mathcal{O}}=\overline{B_{K}}$ as

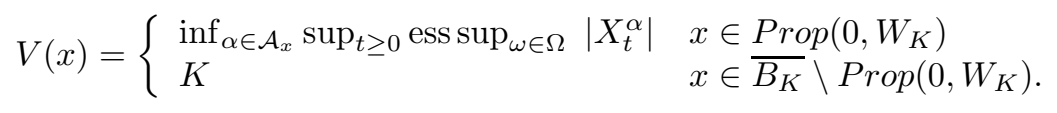

First of all we observe that the function $V$ is well defined: by definition of a.s. stabilizability, there exists a function $\gamma \in \mathcal{K}$ such that $V(x) \leq \gamma(|x|)$. From this we get also that $V$ is continuous at the origin. We observe also that $\operatorname{Prop}\left(0, W_{K}\right) \supseteq B\left(0, \gamma^{-1}(K)\right)$. Moreover $V$ is positive definite. Indeed if $V(x)=0$ then for every $\varepsilon>0$ there exists $\alpha_{\varepsilon}$ such that the corresponding trajectory satisfies $\left|X_{t}\right| \leq \varepsilon$ for all $t \geq 0$ almost surely: so $\inf _{\alpha, \in \mathcal{A}_{x}} \mathbf{E}_{0} \int_{0}^{+\infty}\left|X_{t}\right| e^{-\lambda t} d t=0$ for any fixed $\lambda>0$. By Theorem 1 the inf is attained, and the minimizing control produces a trajectory satisfying a.s. $\left|X_{t}\right|=0$ for all $t \geq 0$. 
To prove the semicontinuity and the differential inequality, we provide another characterization of $V$. We prove that it coincides with

$$
w(x):=\inf \left\{r\left|\exists \bar{\alpha} \in \mathcal{A}_{x}\right| \bar{X}_{t} \mid \leq r \quad \forall t \geq 0 \quad \text { a.s. }\right\} \wedge K .
$$

First of all with the usual argument based on Theorem 1 we get that this infimum is actually a minimum. Then we fix $K \geq k>w(x)$ : by definition there exists $\bar{\alpha} \in \mathcal{A}_{x}$ such that $\left|\bar{X}_{t}\right| \leq k-\varepsilon$ for all $t \geq 0$ a.s. This means that $\sup _{t \geq 0} \operatorname{ess} \sup _{\omega \in \Omega}\left|\bar{X}_{t}\right|<k$ from which we deduce $w(x) \geq V(x)$. The converse is similar.

Therefore, for every $0 \leq \mu<K$, the sublevel set $\{x \in \mathcal{O} \mid V(x) \leq \mu\}$ coincides with $\{x \in$ $\mathbb{R}^{N}\left|\exists \bar{\alpha} \in \mathcal{A}_{x}\right| \bar{X}_{t} \mid \leq \mu \quad \forall t \geq 0$ a.s. $\}$; in particular this gives that the function $V$ is proper. As at the beginning of the proof, we can characterize these sets as the propagation sets of the minima of viscosity supersolutions of suitable Hamilton-Jacobi-Bellman equations. Then, by the results in [7, these sets are closed in $\mathbb{R}^{N}$ and viable with respect to (CSDE). So the function $V$ is LSC. Moreover, by Lemma 11] it satisfies the infinitesimal decrease condition (8).

To prove (ii), we observe that for every $K>0$ we can repeat the previous construction, since the estimate (15) holds in the whole space with $\gamma \in \mathcal{K}_{\infty}$. So we get an increasing sequence of Lyapunov functions $V_{K}$ : for every $K>0$ we construct as before the function $V_{K}$ in the ball $B_{K}$ and extend it to the whole space in the obvious way. Hence the global Lyapunov function for the system is

$$
V(x)=\lim _{K \rightarrow+\infty} V_{K}(x)=\sup _{K>0} V_{K}(x)=\inf _{\alpha \in \mathcal{A}_{x}} \sup _{t \geq 0} \operatorname{ess} \sup _{\omega \in \Omega}\left|X_{t}^{\alpha}\right| .
$$

It is immediate to check that it satisfies the condition (i),(ii),(iii) in the Definition 7 Moreover the proof of the fact that $V$ satisfies the differential condition (iv) relies on standard stability properties of viscosity supersolutions with respect to the pointwise increasing convergence (4]).

Now we prove the converse Lyapunov theorem for asymptotic stability in a bounded set.

Theorem 14 (a.s. asymptotic stabilizability). Assume (3), (4). If the system (CSDE) is a.s. asymptotically stabilizable at the origin in the ball $B_{K}$, then there exist an open set $\mathcal{O}$ containing the origin and a Lipschitz continuous positive definite function $l: \mathcal{O} \rightarrow \mathbb{R}$ such that

$$
V(x)=\inf _{\alpha \in \mathcal{A}_{x}} e s s \sup _{\omega \in \Omega} \int_{0}^{+\infty} l\left(X_{t}^{\alpha}\right) d t
$$

is a Lyapunov function for the system in $\mathcal{O}$.

Proof. The estimate (77) permits to construct a positive definite Lipschitz continuous function $l: \mathbb{R}^{N} \rightarrow \mathbb{R}$ as follows. $t_{x}(U)$ denotes the random time spent in the set $U$ by the trajectory $\bar{X}$. Using the properties of the function $\beta$ we get that, for every $r \leq K$ and $x \in B_{K}$, the time $t_{x}\left(\mathbb{R}^{N} \backslash B_{r}\right)(\omega)$ spent by the trajectory $\bar{X}$ outside $B_{r}$ is almost surely bounded and moreover it satisfies

$$
\sup _{x \in B_{K}} \operatorname{ess} \sup _{\omega \in \Omega} t_{x}\left(\mathbb{R}^{N} \backslash B_{r}\right)(\omega)<+\infty .
$$

We consider now a decreasing sequence of positive numbers $r_{i}$ such that $r_{0}<K$ and $\lim _{i \rightarrow+\infty} r_{i}=$ 0 . We define

$$
T_{i}=\sup _{x \in B_{K}} \operatorname{ess} \sup _{\omega \in \Omega} t_{x}\left(\mathbb{R}^{N} \backslash B_{r_{i}}\right)(\omega) .
$$

The sequence of positive numbers $T_{i}$ is increasing as $i \rightarrow+\infty$ : we can choose a decreasing sequence of positive numbers $l_{i}$ such that $\sum_{i=0}^{+\infty} l_{i} T_{i}=M<+\infty$. The function $l: \mathbb{R}^{N} \rightarrow \mathbb{R}$ is therefore defined as a radial Lipschitz continuous, positive definite, nondecreasing function which satisfies $l(0)=0, l(|x|)=l_{i+1}$ for $|x|=r_{i}$ and $l(|x|)=l_{1}$ for every $|x| \geq r_{0}$.

The candidate Lyapunov function is

$$
V(x)=\inf _{\alpha \in \mathcal{A}_{x}} \operatorname{ess} \sup _{\omega \in \Omega} \int_{0}^{+\infty} l\left(\left|X_{t}^{\alpha}\right|\right) d t \quad \text { for } x \in B_{K} .
$$


The rest of the proof will be devoted to show that this function satisfies the properties of Definition 7 and then is a strict Lyapunov function. First of all $V$ is well defined:

$$
V(x)=\inf _{\alpha \in \mathcal{A}_{x}} \operatorname{ess} \sup _{\omega \in \Omega} \int_{0}^{+\infty} l\left(\left|X_{t}^{\alpha}\right|\right) d t \leq \operatorname{ess} \sup _{\omega \in \Omega} \int_{0}^{+\infty} l\left(\left|\bar{X}_{t}\right|\right) d t \leq \sum_{i=0}^{+\infty} l_{i} T_{i}=M .
$$

By definition $V(x) \geq 0$ for every $x$ and $V(0)=0$. We assume now that for some $x \neq 0 V(x)=0$ : this means that for every $\varepsilon>0$ there exists $\alpha_{\varepsilon} \in \mathcal{A}_{x}$ such that $\int_{0}^{+\infty} l\left(\left|X_{t}^{\alpha_{\varepsilon}}\right|\right) d t \leq \varepsilon$ almost surely. Then $\inf _{\alpha} \mathbf{E}_{x} \int_{0}^{+\infty} l\left(\left|X_{t}^{\alpha}\right|\right) d t=0$ : from this, by the usual argument based on Theorem 1 we deduce that $x=0$. We show now that the function $V$ is continuous at the origin. Recalling the definition of $t_{x}\left(\mathbb{R}^{N} \backslash B_{r}\right)$ and the a.s. asymptotic stabilizability, we get that $t_{x}\left(\mathbb{R}^{N} \backslash B_{r}\right)=0$ almost surely for initial data $x$ such that $\beta(|x|, 0) \leq r$. Therefore, by the continuity at $r=0$ of the function $\beta(r, 0)$, for every $\varepsilon>0$ there exists $\delta>0$ such that, for $|x| \leq \delta, \beta(|x|, 0) \leq \varepsilon$ and then $t_{x}\left(\mathbb{R}^{N} \backslash B_{k}\right)=0$ almost surely for every $k \geq \varepsilon$. So we get $V(x) \leq \sum_{i=i(\varepsilon)}^{+\infty} l_{i} T_{i}$ where $i(\varepsilon)$ is the minimum index for which $r_{i(\varepsilon)} \leq \varepsilon$. Since the sum $\sum_{i} l_{i} T_{i}$ converges and $r_{i} \rightarrow 0$ as $i \rightarrow+\infty$, for every $\theta>0$, we can choose $\varepsilon>0$ such that $\sum_{i=i(\varepsilon)}^{+\infty} l_{i} T_{i} \leq \theta$ : this gives the continuity at the equilibrium. To conclude the proof we have to provide another equivalent definition of $V$. We consider the new system in $\mathbb{R}^{N+1}$

$$
(C S D E 2)\left\{\begin{array}{l}
d\left(X_{t}, Y_{t}\right)=\bar{f}\left(X_{t}, Y_{t}, \alpha_{t}\right) d t+\bar{\sigma}\left(X_{t}, Y_{t}, \alpha_{t}\right) d\left(B_{t}, 0\right), t>0, \\
\left(X_{0}, Y_{0}\right)=(x, y) .
\end{array}\right.
$$

where $\bar{f}(x, y, \alpha)=(f(x, \alpha), l(x))$ and $\bar{\sigma}(x, y, \alpha)=(\sigma(x, \alpha), 0)$. It satisfies conditions (3) and (4) and has $(0,0)$ as a controlled equilibrium. We introduce now the following function

$$
\begin{aligned}
& W(x, y)=\inf \left\{r\left|\exists \alpha \in \mathcal{A}_{x}\right| Y_{t}^{\alpha} \mid \leq r \forall t \geq 0 \text { a.s. }\right\}= \\
& =\inf \left\{r\left|\exists \alpha \in \mathcal{A}_{x}\right| y+\int_{0}^{+\infty} l\left(\left|X_{t}^{\alpha}\right|\right) d t \mid \leq r \text { a.s. }\right\} .
\end{aligned}
$$

Using Theorem 10 it can be proved easily that this infimum is actually a minimum. So the sublevel sets of $W$ are

$$
\{(x, y) \mid W(x, y) \leq \mu\}=\left\{(x, y)\left|\exists \alpha \in \mathcal{A}_{x}\right| y+\int_{0}^{+\infty} l\left(\left|X_{t}^{\alpha}\right|\right) d t \mid \leq \mu \text { a.s. }\right\} .
$$

Repeating the argument in the proof of Theorem 13 we get that these sets are closed and viable with respect to (CSDE2). So the function $W(x, y)$ is LSC and satisfies, by Lemma 11] in viscosity sense

$$
\sup _{\sigma(x, \alpha) \cdot D_{x} W=0}\left\{-D_{x} W \cdot f(x, \alpha)-\operatorname{trace}\left[a(x, \alpha) D_{x x}^{2} W\right]\right\}-l(|x|) D_{y} W \geq 0 .
$$

Now we show that the candidate Lyapunov function $V$ coincides with the function $W$ on the set $B_{K} \times\{y=0\}$. Assume that $V(x) \leq r$. For every $\varepsilon>0$, there exists $\alpha_{\varepsilon} \in \mathcal{A}_{x}$ such that almost surely $\int_{0}^{+\infty} l\left(\left|X_{t}^{\alpha_{\varepsilon}}\right|\right) d t \leq r+\varepsilon$. Therefore $W(x, 0) \leq r+\varepsilon$ and so we conclude by the arbitrariness of $\varepsilon$. The proof of the opposite inequality $V(x) \leq W(x, 0)$ is similar. From this characterization of the function $V$ we deduce immediately that it is LSC and bounded in $B_{K}$. It remains to check the differential condition (9). We fix $x \neq 0$ in $B_{K}$ and consider $(p, Y) \in \mathcal{J}^{2,-} V(x)$ : by definition, for every $x^{\prime} \rightarrow x$, we get $W\left(x^{\prime}, 0\right) \geq W(x, 0)+p \cdot\left(x^{\prime}-x\right)+1 / 2\left(x^{\prime}-x\right) Y\left(x^{\prime}-x\right)+o\left(\left|x-x^{\prime}\right|\right)$. Using the definition, it is immediate to check $W\left(x^{\prime}, 0\right) \leq W\left(x^{\prime}, y\right)-y$. This implies that if $(p, Y) \in \mathcal{J}^{2,-} V(x)$ then $(p, Y, 1) \in \mathcal{J}^{2,1,-} W(x, 0)$. So the differential condition (9) comes from (13).

\section{$6 \quad$ Extensions}

We can extend the results to the characterization of stabilizability of a closed set $M \subseteq \mathbb{R}^{N}$. We denote with $d(x, M)$ the distance between $x \in \mathbb{R}^{N}$ and $M$. 
Definition 15 (a.s. stabilizability at $M$ ). The system (CSDE) is almost surely (open loop) stabilizable at $M$ if there exists $\gamma \in \mathcal{K}$ such that, for every $x$ in a neighborhood of $M$, there is an admissible control function $\bar{\alpha} . \in \mathcal{A}_{x}$ whose trajectory $\bar{X}$. verifies

$$
d\left(\bar{X}_{t}, M\right) \leq \gamma(d(x, M)) \quad \forall t \geq 0 \quad \text { almost surely. }
$$

Remark 16. From this definition, using Theorem 1 we can deduce that if $M$ is a.s. stabilizable, then it is viable for $(C S D E)$.

We adapt the definition of control Lyapunov function to the case the equilibrium is a set $M$ :

Definition 17 (control Lyapunov functions at $M$ ). Let $\mathcal{O}$ be an open neighborhood of the closed set $M$. A function $V: \mathcal{O} \rightarrow[0,+\infty)$ is a control Lyapunov function at $M$ for $(C S D E)$ if (i) $V$ is lower semicontinuous;

(ii) there exist $\gamma_{1}, \gamma_{2} \in \mathcal{K}$ such that $\gamma_{2}(d(x, M)) \leq V(x) \leq \gamma_{1}(d(x, M))$ for all $x \in \mathcal{O}$;

(iii) for all $x \in \mathcal{O} \backslash M$ and $(p, Y) \in \mathcal{J}^{2,-} V(x)$ there exists $\bar{\alpha} \in A$ such that condition (2) holds.

Theorem 18. Assume (3), (4). Then the system (CSDE) is almost surely stabilizable at $M$ if and only if there exists a control Lyapunov function $V$ at $M$.

\section{References}

[1] J.P. Aubin, G. Da Prato: Stochastic Lyapunov method, NoDEA Nonlinear Differential Equations Appl. 2 (1995), 511-525.

[2] J.P. Aubin, G. Da Prato: The viability theorem for stochastic differential inclusions, Stochastic Anal. Appl. 16 (1998), 1-15.

[3] A. Bacciotti, L. Rosier: Liapunov functions and stability in control theory, Springer, London, 2001.

[4] M. Bardi, I. Capuzzo-Dolcetta: Optimal control and viscosity solutions of Hamilton-JacobiBellman equations, Birkäuser, Boston, 1997.

[5] M. Bardi, A. Cesaroni: Viscosity Lyapunov functions for almost sure stability of degenerate diffusions, in "Elliptic and Parabolic problems, Rolduc and Gaeta 2001", J.Bemelmans et al.eds., pp.322-331, World Scientific, Singapore, 2002.

[6] M. Bardi, A. Cesaroni: Almost sure stabilizability of controlled degenerate diffusions, to appear in SIAM J. Control Optim.

[7] M. Bardi, F. Da Lio: Propagation of maxima and strong maximum principle for viscosity solutions of degenerate elliptic equations. II. Concave operators, Indiana Univ. Math. J. 52 (2003), no. 3, 607-627.

[8] M. Bardi, P. Goatin: Invariant sets for controlled degenerate diffusions: a viscosity solutions approach, in "Stochastic Analysis, Control, Optimization and Applications: A Volume in Honor of W.H. Fleming", W.M. McEneaney, G.G. Yin and Q. Zhang eds., pp. 191-208, Birkhäuser, Boston, 1999.

[9] M. Bardi, R. Jensen: A geometric characterization of viable sets for controlled degenerate diffusions, Set-Valued Anal. 10 (2002), no. 2-3, 129-141.

[10] E.N. Barron, P. Cardaliaguet, R. Jensen:Conditional essential suprema with applications, Appl. Math. Optim. 48 (2003), no. 3, 229-253.

[11] A. Cesaroni: Lyapunov stabilizability of controlled diffusions via a superoptimality principle for viscosity solutions, preprint, submitted. 
[12] A. Cesaroni: Stability properties of controlled diffusion processes via viscosity methods, Ph.D. thesis, University of Padova, 2004.

[13] M.C. Crandall, H. Ishii, P.L. Lions: User's guide to viscosity solutions of second order partial differential equations, Bull. Amer. Math. Soc. 27 (1992), 1-67.

[14] G. Da Prato, H. Frankowska:Invariance of stochastic control systems with deterministic arguments, J. Differential Equations 200 (2004), no. 1, 18-52.

[15] W.H. Fleming, H.M. Soner: Controlled Markov Process and Viscosity Solutions, SpringerVerlag, New York, 1993.

[16] R.A. Freeman, P.V. Kokotovic:Robust nonlinear control design. State-space and Lyapunov techniques, Birkäuser, Boston, 1996.

[17] R.Z. Has'minskii: Stochastic stability of differential equations, Sjithoff and Noordhoff International Publishers, 1980.

[18] U.G. Haussmann and J.P. Lepeltier, On the existence of optimal controls, SIAM J. Control Optim. 28 (1990), 851-902.

[19] H.J. Kushner: Stochastic stability and control, Academic Press, New York, 1967.

[20] H.J. Kushner: Converse theorems for stochastic Liapunov functions, SIAM J. Control Optim. 5 (1967), 228-233.

[21] X. Mao: Exponential stability of stochastic differential equations, Marcel Dekker, New York, 1994.

[22] P.-L. Lions: Optimal control of diffusion processes and Hamilton-Jacobi-Bellman equations. Part 1: The dynamic programming principle and applications, Part 2: Viscosity solutions and uniqueness, Comm. Partial Differential Equations 8 (1983), 1101-1174 and 1229-1276.

[23] L. Rifford: Existence of Lipschitz and semiconcave control-Lyapunov functions, SIAM J. Control Optim. 39 (2000), no. 4, 1043-1064.

[24] H.M. Soner, N. Touzi: Stochastic target problems, dynamic programming, and viscosity solutions, SIAM J. Control Optim. 41 (2002), no. 2, 404-424.

[25] H.M. Soner, N. Touzi: A stochastic representation for mean curvature type geometric flows, Ann. Probab. 31 (2003), no. 3, 1145-1165.

[26] E.D. Sontag: A Lyapunov-like characterization of asymptotic controllability, SIAM J. Control Optim. 21 (1983), no. 3, 462-471.

[27] E.D. Sontag, H.J. Sussmann: Non smooth control Lyapunov functions, Proc. IEEE Conf. Decision and Control, New Orleans, Dec 1995, IEE Publications, 1995.

[28] P. Soravia: Stability of dynamical systems with competitive controls: the degenerate case, J. Math. Anal. Appl. 191 (1995), 428-449.

[29] P. Soravia: Feedback stabilization and $\mathcal{H}_{\infty}$ control of nonlinear systems affected by disturbances: the differential games approach, Dynamics, bifurcations, and control (Kloster Irsee, 2001), 173-190, Lecture Notes in Control and Inform. Sci., 273, Springer, Berlin, 2002. 